\title{
Mutations in PLOD3, encoding lysyl hydroxylase 3, cause a complex connective tissue disorder including recessive dystrophic epidermolysis bullosa-like blistering phenotype with abnormal anchoring fibrils and type VII collagen deficiency
}

Vahidnezhad, Hassan ; Youssefian, Leila ; Saeidian, Amir Hossein ; Touati, Andrew ; Pajouhanfar, Sara ; Baghdadi, Taghi ; Shadmehri, Azam Ahmadi ; Giunta, Cecilia ; Kraenzlin, Marius ; Syx, Delfien ; Malfait, Fransiska ; Has, Cristina ; Lwin, Su M ; Karamzadeh, Razieh ; Liu, Lu ; Guy, Alyson ; Hamid, Mohammad ; Kariminejad, Ariana ; Zeinali, Sirous ; McGrath, John A ; Uitto, Jouni

\begin{abstract}
Epidermolysis bullosa (EB), the paradigm of heritable skin fragility disorders, is associated with mutations in as many as 20 distinct genes. One of the clinical variants, recessive dystrophic EB (RDEB), demonstrates sub-lamina densa blistering accompanied by alterations in anchoring fibrils due to mutations in COL7A1. In this study, we characterized a patient with widespread connective tissue abnormalities, including skin blistering similar to that in RDEB. Whole exome sequencing, combined with genome-wide homozygosity mapping, identified a homozygous missense mutation in PLOD3 encoding lysyl hydroxylase 3 (LH3). No mutations in COL7A1, the gene previously associated with RDEB, were detected. The level of LH3 was dramatically reduced in the skin and fibroblast cultures from the patient. The blistering in the skin occurred below the lamina densa and was associated with variable density and morphology of anchoring fibrils. The level of type VII collagen expression in the skin was markedly reduced. Analysis of hydroxylysine and its glycosylated derivatives (galactosyl-hydroxylysine and glucosyl-galactosyl-hydroxylysine) revealed marked reduction in glycosylated hydroxylysine. Collectively, these findings indicate that PLOD3 mutations can result in a dystrophic EB-like phenotype in the spectrum of connective tissue disorders and add it to the list of candidate genes associated with skin fragility.
\end{abstract}

DOI: https://doi.org/10.1016/j.matbio.2018.11.006

Posted at the Zurich Open Repository and Archive, University of Zurich ZORA URL: https://doi.org/10.5167/uzh-160756

Journal Article

Accepted Version

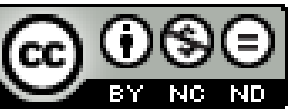

The following work is licensed under a Creative Commons: Attribution-NonCommercial-NoDerivatives 4.0 International (CC BY-NC-ND 4.0) License.

Originally published at:

Vahidnezhad, Hassan; Youssefian, Leila; Saeidian, Amir Hossein; Touati, Andrew; Pajouhanfar, Sara; Baghdadi, Taghi; Shadmehri, Azam Ahmadi; Giunta, Cecilia; Kraenzlin, Marius; Syx, Delfien; Malfait, 
Fransiska; Has, Cristina; Lwin, Su M; Karamzadeh, Razieh; Liu, Lu; Guy, Alyson; Hamid, Mohammad; Kariminejad, Ariana; Zeinali, Sirous; McGrath, John A; Uitto, Jouni (2019). Mutations in PLOD3, encoding lysyl hydroxylase 3, cause a complex connective tissue disorder including recessive dystrophic epidermolysis bullosa-like blistering phenotype with abnormal anchoring fibrils and type VII collagen deficiency. Matrix Biology, 81:91-106.

DOI: https://doi.org/10.1016/j.matbio.2018.11.006 


\section{Accepted Manuscript}

Mutations in PLOD3, encoding lysyl hydroxylase 3, cause a complex connective tissue disorder including recessive dystrophic epidermolysis bullosa-like blistering phenotype with abnormal anchoring fibrils and type VII collagen deficiency

Hassan Vahidnezhad, Leila Youssefian, Amir Hossein Saeidian, Andrew Touati, Sara Pajouhanfar, Taghi Baghdadi, Azam Ahmadi Shadmehri, Cecilia Giunta, Marius Kraenzlin, Delfien Syx,

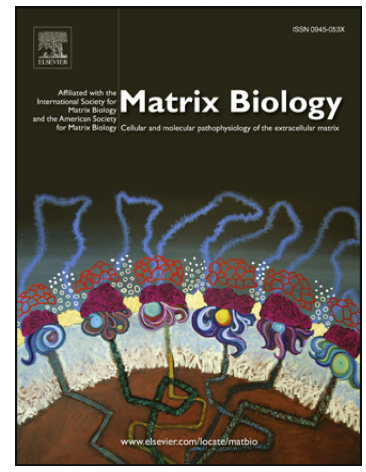
Fransiska Malfait, Cristina Has, Su M. Lwin, Razieh Karamzadeh, Lu Liu, Alyson Guy, Mohammad Hamid, Ariana Kariminejad, Sirous Zeinali, John A. McGrath, Jouni Uitto

PII: S0945-053X(18)30345-7

DOI:

Reference: https://doi.org/10.1016/j.matbio.2018.11.006

To appear in: MATBIO 1542

Received date: Matrix Biology

Revised date: 7 August 2018

Accepted date:

16 November 2018

16 November 2018

Please cite this article as: Hassan Vahidnezhad, Leila Youssefian, Amir Hossein Saeidian, Andrew Touati, Sara Pajouhanfar, Taghi Baghdadi, Azam Ahmadi Shadmehri, Cecilia Giunta, Marius Kraenzlin, Delfien Syx, Fransiska Malfait, Cristina Has, Su M. Lwin, Razieh Karamzadeh, Lu Liu, Alyson Guy, Mohammad Hamid, Ariana Kariminejad, Sirous Zeinali, John A. McGrath, Jouni Uitto , Mutations in PLOD3, encoding lysyl hydroxylase 3 , cause a complex connective tissue disorder including recessive dystrophic epidermolysis bullosa-like blistering phenotype with abnormal anchoring fibrils and type VII collagen deficiency. Matbio (2018), https://doi.org/10.1016/j.matbio.2018.11.006

This is a PDF file of an unedited manuscript that has been accepted for publication. As a service to our customers we are providing this early version of the manuscript. The manuscript will undergo copyediting, typesetting, and review of the resulting proof before it is published in its final form. Please note that during the production process errors may be discovered which could affect the content, and all legal disclaimers that apply to the journal pertain. 


\section{Mutations in PLOD3, encoding lysyl hydroxylase 3, cause a complex}

\section{connective tissue disorder including recessive dystrophic epidermolysis}

\section{bullosa-like blistering phenotype with abnormal anchoring fibrils and type}

\section{VII collagen deficiency}

Hassan Vahidnezhad ${ }^{\mathrm{a}, \mathrm{b}, \#}$, Leila Youssefian ${ }^{\mathrm{b}, \mathrm{c}, \mathrm{d} \#}$, Amir Hossein Saeidian ${ }^{\mathrm{b}, \mathrm{d}}$, Andrew Touati ${ }^{\mathrm{b}, \mathrm{e}}$, Sara Pajouhanfar $^{\mathrm{b}}$, Taghi Baghdadi ${ }^{\mathrm{f}}$, Azam Ahmadi Shadmehri ${ }^{\mathrm{g}}$, Cecilia Giunta ${ }^{\mathrm{h}}$, Marius Kraenzlin ${ }^{\mathrm{i}}$, Delfien Syx ${ }^{\mathrm{j}}$, Fransiska Malfait ${ }^{\mathrm{j}}$, Cristina Has ${ }^{\mathrm{k}}$, Su M. Lwin ${ }^{1}$, Razieh Karamzadeh $^{\mathrm{m}, \mathrm{n}}$, Lu Liu ${ }^{\mathrm{o}}$, Alyson Guy ${ }^{1}$, Mohammad Hamid ${ }^{\mathrm{a}}$, Ariana Kariminejad ${ }^{\mathrm{p}}$, Sirous Zeinali ${ }^{\mathrm{a}, \mathrm{q}}$, John A. McGrath ${ }^{\text {', and }}$ Jouni Uitto ${ }^{\mathrm{b}, \mathrm{r}}$

${ }^{a}$ Molecular Medicine Department, Biotechnology Research Center, Pasteur Institute of Iran, Tehran, Iran;

${ }^{\mathrm{b}}$ Department of Dermatology and Cutaneous Biology, Sidney Kimmel Medical College, Thomas Jefferson University, Philadelphia, Pennsylvania, USA;

${ }^{\mathrm{c}}$ Department of Medical Genetics, School of Medicine, Tehran University of Medical Sciences, Tehran, Iran;

${ }^{\mathrm{d}}$ Genetics, Genomics and Cancer Biology PhD Program, Thomas Jefferson University, Philadelphia, Pennsylvania, USA;

${ }^{\mathrm{e}}$ Drexel University College of Medicine, Philadelphia, Pennsylvania, USA;

${ }^{\mathrm{f}}$ Department of Orthopedic Surgery, Imam Khomeini Hospital, Tehran University of Medical Sciences, Tehran, Iran;

${ }^{\mathrm{g}}$ Department of Genetics, Fars Science and Research Branch, Islamic Azad University, Marodasht, Iran;

h Division of Metabolism, Connective Tissue Unit and Research, Children's Center, University Children's Hospital Zurich, Zurich, Switzerland;

${ }^{\mathrm{i}}$ Medical Faculty of the University of Basel, and Clinic for Endocrinology, Diabetes \& Metabolism, University Hospital Basel, Basel, Switzerland;

${ }^{\mathrm{j}}$ Center for Medical Genetics, Ghent University Hospital, 9000 Ghent, Belgium;

${ }^{\mathrm{k}}$ Department of Dermatology, Medical Center - University of Freiburg, Faculty of Medicine, University of Freiburg, Germany;

${ }^{1}$ St John's Institute of Dermatology, King's College London, Guy's Campus, London;

minstitute of Biochemistry and Biophysics, University of Tehran, Tehran, Iran;

${ }^{\mathrm{n}}$ Department of Molecular Systems Biology, Cell Sciences Research Center, Royan Institute for Stem Cell Biology and Technology, ACECR, Tehran, Iran; 'Viapath, St Thomas' Hospital, London, UK;

${ }^{\mathrm{p}}$ Kariminejad-Najmabadi Pathology \& Genetics Center, Tehran, Iran; 
${ }^{\mathrm{q}}$ Kawsar Human Genetics Research Center, Tehran, Iran;

${ }^{\mathrm{rq}}$ Jefferson Institute of Molecular Medicine, Thomas Jefferson University, Philadelphia, Pennsylvania, USA;

\#These authors contributed equally to this work.

Running title: PLOD3 mutations cause EB

Abbreviations: EB, epidermolysis bullosa; RDEB, recessive dystrophic EB; GHL, galactosyl-

hydroxylysine; GGHL, glucosyl-galactosyl-hydroxylysine; ROH, region of homozygosity; WES, whole exome sequencing

Address for the Corresponding Author:

Jouni Uitto, MD, PhD

Department of Dermatology and Cutaneous Biology

Sidney Kimmel Medical College at Thomas Jefferson University

233 South $10^{\text {th }}$ Street, Suite 450 BLSB

Philadelphia, PA 19107, USA

Tel: 215-503-5785; Fax: 215-503-5788

E-mail: Jouni.Uitto@jefferson.edu 


\begin{abstract}
Epidermolysis bullosa (EB), the paradigm of heritable skin fragility disorders, is associated with mutations in as many as 20 distinct genes. One of the clinical variants, recessive dystrophic EB (RDEB), demonstrates sub-lamina densa blistering accompanied by alterations in anchoring fibrils due to mutations in COL7A1. In this study, we characterized a patient with widespread connective tissue abnormalities including skin blistering similar to that in RDEB. Whole exome sequencing, combined with genome-wide homozygosity mapping, identified a homozygous missense mutation in PLOD3 encoding lysyl hydroxylase 3 (LH3). No mutations in COL7A1, the gene previously associated with RDEB, were detected. The level of LH3 was dramatically reduced in the skin and fibroblast cultures from the patient. The blistering in the skin occurred below the lamina densa and was associated with variable density and morphology of anchoring fibrils. The level of type VII collagen expression in the skin was markedly reduced. Analysis of hydroxylysine and its glycosylated derivatives (galactosyl-hydroxylysine and glucosylgalactosyl-hydroxylysine) revealed marked reduction in glycosylated hydroxylysine. Collectively, these findings indicate that PLOD3 mutations can result in a dystrophic EB-like phenotype in the spectrum of connective tissue disorders and add it to the list of candidate genes associated with skin fragility.
\end{abstract}

Keywords: Epidermolysis bullosa; lysyl hydroxylase 3; PLOD3, type VII collagen, collagen glycosylation 


\section{Introduction}

\section{Phenotypic and genetic heterogeneity of EB}

Epidermolysis bullosa (EB), a group of heritable skin fragility disorders, manifests with blistering and erosions leading to ulcers with poor healing tendency [1]. EB is divided into four broad categories based on the level of tissue separation within the dermal-epidermal basement membrane zone as a result of external trauma. These different subtypes of EB demonstrate extensive phenotypic variability, which, to a large part, reflects genetic heterogeneity, and as many as 20 distinct genes have so far been shown to be associated with different forms of EB [2$5]$.

One form of EB is the recessive dystrophic EB (RDEB), which in most severe cases manifests with tense blisters which can develop into non-healing ulcers prone to infections and later in life lead to development of aggressive squamous cell carcinomas resulting in early demise $[1,6]$. Tissue separation in the dystrophic form of EB is below the lamina densa within the upper papillary dermis at the level of anchoring fibrils. Anchoring fibrils, which consist of type VII collagen, can be reduced in number, morphologically altered or entirely absent in RDEB as a result of mutations in the COL7A1 gene which encodes the type VII collagen precursor polypeptides [7]. Thus far, COL7Al is the only gene that has been implicated in the pathobiology of dystrophic forms of EB.

\section{Type VII collagen and the anchoring fibrils}

Type VII collagen is initially synthesized by basal keratinocytes and dermal fibroblasts as a 290kDa polypeptide, and three of these chains assemble intracellularly into a triplex molecule 
through association of their carboxy-terminal ends, followed by folding of the collagenous domain into stable triple-helical conformation at physiological body temperatures in a process requiring prolyl-4-hydroxylation [7]. In addition, a select number of lysyl residues are hydroxylated to form hydroxylysyl residues, and some of them are subsequently glycosylated in a step wise fashion to form either galactosyl-hydroxylysine (GHL) or glucosyl-galactosylhydroxylysine (GGHL) residues. Following intracellular processing, type VII collagen molecules are secreted into the extracellular space where they assemble into a centro-symmetric dimer arrangement with tail-to-tail interactions through their carboxy-termini [8]. Subsequently, a large number of such dimer molecules laterally aggregate to form anchoring fibrils which associate through their amino-terminal ends with the components of the lamina densa to form Ushaped structures which entrap type I collagen molecules within the upper papillary dermis [9]. Thus, the anchoring fibrils tether the dermal-epidermal basement membrane to the underlying dermis.

\section{Post-translational modification of collagens}

Hydroxylation of lysyl residues to form hydroxylysine residues is an essential post-translational modification catalyzed by lysyl hydroxylases (LH), a family of four different isoforms: LH1, LH2a, LH2b and LH3 (the latter one also known as procollagen-lysine, 2-oxoglutarate 5dioxygenase 3; PLOD3) [10]. Subsequently, certain hydroxylysine residues in the Y position of the repeating gly-X-Y sequence of collagen polypeptides become O-glycosylated in the intracellular milieu classically by two separate enzymatic activities, collagen galactosyltransferase and collagen glucosyltransferase which sequentially transfer UDPgalactose to peptidyl hydroxylysine and UDP-glucose to galactosyl-hydroxylysine residues, 
respectively [11]. Interestingly, however, in addition to its ability to hydroxylate lysyl residues, LH3 is also able to glycosylate hydroxylysine residues. LH3 is also the only LH which is secreted and present in the extracellular space $[12,13]$. Recent studies utilizing immune coprecipitation and Western blotting analyses as well as in situ proximity ligation assay have demonstrated a direct interaction between LH3 and type VII collagen [14]. Immunoelectron microscopy and morphometric measurements utilizing antibodies raised against the amino- and carboxy-termini of type VII collagen as well as the amino-terminus of LH3 have demonstrated immune labeling beneath the epidermis where LH3 was shown to be localized to two main compartments: (a) over the lamina densa/basement membrane directly beneath hemidesmosomes, and (b) in the sub-lamina densa region in the area corresponding to the central collagenous domain of type VII collagen [14]. However, whether LH3 is catalytically active in these extracellular sites and whether it catalyzes hydroxylation and subsequent glycosylation of lysyl residues in type VII collagen is currently unknown.

The interest to study LH3 in EB, in addition to its co-localization with type VII collagen at the dermal-epidermal junction, was increased by demonstration that the level of LH3 is significantly reduced in RDEB patients' skin and in keratinocytes derived from these patients [14]. Conversely, overexpression of type VII collagen in RDEB cells resulted in increased LH3 secretion, suggesting functional inter-relationship between LH3 and type VII collagen.

\section{LH3 deficiency leads to widespread connective tissue abnormalities}

Considering the fact that LH3 potentially participates in post-translational modification of all collagens, deficiency in this protein could be predicted to result in widespread connective tissue disorders. This, in fact, has been demonstrated in Plod3 knock-out mice which demonstrate 
connective tissue perturbations, including changes in collagen fibers in the skin [15]. In addition, LH3 deficiency in patients with mutations in PLOD3 has been shown to result in severe morbidity as a result of features of a number of known collagen disorders, including musculoskeletal abnormalities [16].

In this report we describe a patient with biallelic mutations in PLOD3, the gene encoding LH3. The patient demonstrated widespread connective tissue abnormalities, including skin blistering similar to that noted in RDEB, reduced type VII collagen expression, and paucity of anchoring fibrils, features that have not been emphasized in the previous cases. .

\section{RESULTS}

We have explored the molecular basis of EB patients in Iranian, largely consanguineous, families by next generation sequencing approaches, including utilization of a gene-targeted sequencing panel consisting of 21 skin fragility-associated genes as well as by whole exome sequencing (WES) [17-20]. In a consanguineous family, the proband was found to have extensive connective tissue abnormalities, including skin blistering and erosions since birth, consistent with dystrophic forms of EB (Figure 1). The parents were clinically normal, suggesting autosomal recessive inheritance, and there was evidence of consanguinity in the family (Figure 1a).

\section{Case Report}

The proband, a 4.5-year-old male, was born to healthy Iranian Baloch second cousins once removed (Figure 1a). He was referred to our laboratory for genetic testing with an initial diagnosis of Escobar syndrome and epidermolysis bullosa. At the first visit, a slender male, with developmental delay, manifested with musculoskeletal involvement including scoliosis, flexion 
contractions in elbows and carpometacarpal joints, webbing across the second interphalangeal joint of the left hand, prominently enlarged knee and elbow joints, short legs, talipes equinovarus, and reduced muscle mass (Figure 1e-h). He had pterygia of the neck, axilla, antecubital, popliteal, digital and intercrural areas. The thumbs were abnormally folded over palm. Skin creases on both palms were reduced. In addition, trauma-induced skin blisters over the prominent body areas, particularly on elbows, feet, knees, and ears with history of delayed wound healing, were noted (Figure 1b, c). He had dystrophic nails in his feet, but his hair and teeth were normal. The parents claimed that the nails were rapidly growing. His height, weight and head circumference were $96 \mathrm{~cm}\left(<3^{\text {rd }}\right.$ centile $), 10 \mathrm{~kg}\left(<3^{\text {rd }}\right.$ centile $)$ and $46 \mathrm{~cm}\left(<2^{\text {nd }}\right.$ centile $)$, respectively. Ophthalmologic examination showed amblyopia and strabismus, in addition to his neonatal ophthalmologic findings (see below).

The patient was born at term following normal vaginal delivery without complications during pregnancy. His birth weight, height, and head circumference were within the normal range, 2,650 g, $48 \mathrm{~cm}$ and $33 \mathrm{~cm}$, respectively. Milestones were delayed; rolled over at 5 months, sat at 8 months, stood at 2 years, walked at 3 years and started saying his first works at the age of three years. He now at the age of 4.5 years has a vocabulary of 50 words. The patient was born with a right eye cataract, ptosis, severe myopia, flat face profile with a long philtrum, low set ears, and short nose with anteverted nares. Ophthalmologic surgery to correct unilateral ptosis was performed at 10 months of age. There were two complicated pregnancies in the family, one before and one after the proband's birth (Figure 1a). The first one was terminated at 12 weeks due to intrauterine growth failure, and the second one at 18 weeks due to underdevelopment of the neural system. The proband had neonatal hyperbilirubinemia (total bilirubin 19; normal level $<1 \mathrm{mg} / \mathrm{dl}$ ) at the first week of his life, relieved by phototherapy. To rule out kernicterus, brain 
magnetic resonance imaging studies were performed revealing hypersignal areas in basal ganglia favoring kernicterus, as well as another hypersignal region in the left parietal brain suggesting hemorrhage and calcification.

To work up syndromic diseases, plain chest radiography and chest computed tomography (CT) scan were conducted at 1 month of age. The proband had right diaphragmatic eventration seen in spiral CT scan of the thorax without intravenous contrast, and there was a large soft tissue density in medial and anterior basal aspects of right hemithorax with midline and leftward extension, displacing the heart upwards, likely attributed to herniation of hepatic parenchyma into the thorax in the context of a diaphragmatic hernia (Morgagni) or severe eventration (Figure 1d). Only a small part of right lobe of liver was seen in the right subdiaphragmatic space. Diaphragmatic eventration disappeared with aging without intervention. His echocardiography was normal with $60 \%$ ejection fraction at 9 months of age. In addition, the patient had normal electrodiagnostic study in both upper limbs, with no evidence of peripheral nerve lesion or polyneuropathy at 18 months of age. There was normal electromyography in bilateral flexor carpi ulnaris muscles. In comparison with a similar case previously presented [16], our patient did not show any evidence of cutaneous hemorrhage, vascular aneurysms, sensory neural hearing loss or coarse hair, some of which had developed in the previous case at an older age (Table S1).

\section{Whole exome sequencing identifies a pathogenic missense mutation in PLOD3}

Targeted multi-gene sequencing array consisting of 21 skin fragility-associated genes was first applied to DNA from the proband and his parents. No pathogenic mutations in these 21 candidate genes previously associated with EB were found. It should be noted that careful examination of previously reported EB-associated genes, and specifically COL7A1, failed to 
reveal pathogenic mutations, including large deletions. The proband's DNA was subsequently submitted to WES, which identified a total of $~ 134,000$ sequence variants (Figure 2). Following bioinformatics filtering, which considered exonic/splicing variants only, removed synonymous sequence variants, and identified homozygous/compound heterozygous variants with minor allelic frequency $(\mathrm{MAF})<0.001$, reduced the number of annotated, potentially pathogenic variants to 16 (Figure $2 b$ ).

Homozygosity mapping using a single nucleotide polymorphism panel of 640,000 markers identified several regions of homozygosity $(\mathrm{ROH})$, including one of $\sim 45 \mathrm{Mb}$ in the patient's DNA in chromosome 7 (Figure 2a). This homozygosity block was not present in clinically healthy parents. There was no ROH associated with chromosome 3 which harbors the COL7A1 locus in the region 3p21.3. Overlapping of the 16 variants identified by WES with the $45 \mathrm{Mb} \mathrm{ROH}$ reduced the number of potential candidate genes containing homozygous mutations to eight (Table S2). Subsequently, segregation analysis of the variants in the family and phenotypic matching with the known functions of the genes suggested PLOD3 as the candidate gene. Next generation sequencing identified a homozygous mutation in the PLOD3 gene (NM_001084), c.1880T>C; p.Leu627Pro, and this was confirmed by bidirectional Sanger sequencing (Figure 2c). The Leu627 residue is highly conserved through evolution between $\mathrm{H}$. sapiens and D. melanogaster (MutationTaster), and this missense substitution was predicted to be pathogenic by bioinformatics prediction programs (ANNOVAR) [21]. The parents were shown by Sanger sequencing to be heterozygous carriers of the p.Leu627Pro mutation (Figure 2c). Three-dimensional protein modeling localized the p.Leu627Pro mutation to the LH domain of LH3, responsible for lysyl hydroxylase activity which is dependent on the presence of $\mathrm{Fe}^{2+}$ and 2-oxoglutaric acid (Figure 2d). Western blotting of protein synthesized by fibroblasts established 
from the skin of the patient showed markedly reduced, by 80\%, levels of LH3, when compared to three unaffected controls (Figure 2e, f). It should be noted, however, that the PLOD3 mRNA levels in the patient's fibroblasts measured by whole-transcriptome sequencing and confirmed by RT-PCR, were the same as in control cells, indicating post-translational consequences of the mutation on LH3 expression. However, the expression of type VII collagen was reduced significantly. There are no obvious changes in expression of types I, IV, V, and VI collagens (Figure 2g).

\section{Consequences of the $P L O D 3$ mutation at structural level}

Histopathologic examination of the skin adjacent to a blister revealed separation at the dermalepidermal junction, with no evidence of inflammation (Figure 3a). Ultrastructurally, the level of cleavage was shown to be below the lamina densa (Figures 3b). Transmission electron microscopy (TEM) of the patient's skin revealed changes in the lamina densa, and specifically, while in some parts of skin the lamina densa appeared relatively intact, in other areas it was interrupted and fragmented (Figure 4). TEM revealed a variable number and altered morphology of anchoring fibrils in both intact and blistered skin (Figure 5). In areas with no sub-lamina densa cleavage, there were a number of well-formed anchoring fibrils, while in other sections, the dermal-epidermal junction was intact but the sub-lamina densa region was devoid of anchoring fibrils. In some areas, there was extensive sub-lamina densa cleavage with only a few, thin, wisplike structures with no clearly discernable anchoring fibrils present (Figure 5).

Histopathology at low magnification demonstrated flattened appearance of the dermalepidermal junction and dense appearance of the papillary dermis (Figure 6a). Several fibroblasts were found to be present close to the dermal-epidermal junction embedded within the dense, 
homogeneous upper dermis consisting of densely packed individual collagen bundles as observed by histopathology and TEM (Figure 6b, c). Many of the fibroblasts displayed dilated endoplasmic reticulum and prominent vesicles close to the cell membrane, consistent with high biosynthetic activity. Collectively, these findings were suggestive of a blistering disorder with sub-lamina densa tissue separation and fibrosis overlapping with the ultrastructural features of RDEB.

\section{Reduced Expression of LH3 and Type VII Collagen in the Skin}

Immunofluorescence microscopy with an antibody specific for LH3 showed its presence in a punctate pattern at the dermal-epidermal junction in the control skin, while it was completely absent in the patient's skin (Figure 7a). Staining for type VII collagen with a monoclonal antibody, revealed bright linear staining in control skin, while in the patient's skin the staining in the blistered area showed markedly attenuated amounts of type VII collagen, present primarily in the roof of the blister (Figure 7a).

Additional immunostaining experiments with antibodies recognizing type IV and XVII collagens, laminin 332 ( $\gamma 2$-subunit), $\beta 4$ integrin and plectin confirmed that the blistering occurred below the lamina densa (Figure 7b). Staining for keratins 1, 5, 10 and 14 revealed essentially normal epidermal pattern with somewhat attenuated staining (Figure 7c). Thus, antigen mapping established the plane of cleavage below the lamina densa. In addition, the intensity of staining for type XVII collagen was attenuated while the staining for type IV collagen appeared unaltered as compared to control (Figure 6b).

Quantitation of the presence of type VII collagen by Mean Fluorescence Intensity (MFI) assay [22] revealed consistent reduction, yet considerable variability, in the presence of type VII 
collagen in different parts of the skin of the proband. In some areas, the intensity was reduced only by $\sim 30 \%$, while in some areas, type VII collagen was present only at the level of $\sim 23 \%$ of the control (Figure 8). In general, the areas showing the lowest level of expression of type VII collagen were characterized ultrastructurally by a marked reduction or absence of anchoring fibrils (see above).

Collectively, the histopathologic, ultrastructural, expression profile, and immunofluorescence findings in the proband's skin demonstrated features of dystrophic forms of $\mathrm{EB}$, a disease previously associated with mutations in the COL7A1 gene [7]. As indicated above, no pathogenic homozygous or compound heterozygous mutations in COL7AI were identified by EB-gene targeted sequencing panel or WES, suggesting that PLOD3 is the second gene harboring mutations in patients with RDEB-like clinical presentation.

\section{Reduced Glycosylation of Urinary Hydroxylysine}

Since LH3 displays both lysyl hydroxylation and hydroxylysyl glycosylation activities [15], one of the potential consequences of PLOD3 mutations would be reduced hydroxylysine and/or galactosyl-hydroxylysine (GHL) and glucosylgalactosyl hydroxylysine levels (GGHL) in collagens. To explore this possibility, the relative levels of lysine, hydroxylysine, GGHL and GHL in the form of collagen-derived pyridinoline compounds were determined in the urine of the proband and his parents by liquid chromatography. First, quantitation of the ratio of total hydroxylysine/lysine did not reveal differences between the patient and his parents (Figure 9). Furthermore, the ratio in the 4.5-year old patient was within the limits of age-matched controls (3-5 years). However, significantly decreased levels of glycosylated hydroxylysine derivatives were noted in the proband with a concomitant relative increase in hydroxylysine (Figure 9). As a 
consequence, the ratio of (GGHL + GHL)/HL in the proband was reduced to about $17 \%$ of that measured in his parents, a value well below the age-matched controls. Finally, the ratio of GGHL/GHL was unaltered as compared to controls. .

\section{DISCUSSION}

In this study, we have demonstrated sub-lamina densa blistering with markedly reduced type VII collagen in the skin of a patient with a homozygous missense mutation in PLOD3. In addition to skin blistering, the proband demonstrated extensive joint contractures, skeletal abnormalities and reduced growth. Previously, a family with mutations in PLOD3 has been reported, the proband being compound heterozygous for c.668A>G, p.Asn223Ser; c.2071delT, p.Cys691AlafsX9 [16]. These mutations resided in different parts of LH3, responsible for lysyl hydroxylation and hydroxylysine glycosylation activities, respectively (see Figure $2 \mathrm{~d}$ ). The patients in the previous study had an extremely complex phenotype, including deafness, myopia, arterial ruptures, osteopenia, joint contractures, bone fractures, and cataracts, as well as clinically observed skin blistering, apparently reflecting the deficiency in both lysyl hydroxylation and glycosylation of hydroxylysyl residues. In contrast, our patient had a homozygous mutation interfering primarily with the glycosylation activity, which may explain somewhat milder phenotype, although it should be noted that our patient was much younger ( 4.5 years) than those reported previously (up to 14 years of age). Nevertheless, in both cases there were a number of extracutaneous manifestations attesting to the multisystem consequences of the PLOD3 mutations.

Interestingly, an earlier study reported deficiency of galactosylhydroxylysylglucosyltransferase activity in a large Finnish family with autosomal dominant epidermolysis bullosa simplex [23]. Most of the affected members of the family showed low enzymatic activity 
in serum, skin and cultured skin fibroblasts, and there was a markedly reduced urinary excretion of GGHL. This decrease in glucosyltransferase activity was limited to this enzyme, and lysyl hydroxylase and hydroxylysyl galactosyltransferase activities, as determined in skin biopsy specimens, were normal [23]. How the deficiency in galactosylhydroxylysineglucosyltransferase, an enzyme primarily involved in post-translational modification of collagens might cause EB simplex is unclear, but it could serve as a modifier of the severity of EB phenotype [15] as a specific defect in glycosylation of epidermal cell membranes has been reported in EB simplex [24]. Previously, skin blistering has also been reported in patients with extensive pulmonary and renal involvement due to mutations in the ITGA3 gene encoding $\alpha 3$ subunit of the $\alpha 3 \beta 1$ integrin complex [25]. These observations suggest the concept of classification of skin fragility disorders in the spectrum of EB into two distinct categories: syndromic and non-syndromic with prognostic implications [26]. In the latter category, the epithelia of skin and various mucous membranes are primarily affected, while in the first one, the disease manifests in addition to skin in a number of extracutaneous tissues and internal organs in a multisystem fashion.

It should be noted that the mutation, p.Leu627Pro, was shown to reside within the LH domain responsible for lysyl hydroxylation, yet the ratio of urinary hydroxylysine/lysine was not altered. This discrepancy could be explained by the suggestion that this nonsense mutation does not interfere with the catalytic activity of LH3, but rather results in conformational changes predisposing the protein to degradation and loss of both lysyl hydroxylation and hydroxylysyl glycosylation activities. In such case, lysyl hydroxylation could be compensated by other members of the LH family (LH1). At the same time, LH3 has very low, if any, galactosylhydroxylysyl transferase activity while it is the major protein responsible for 
galactosylhydroxylysyl glucosyltransferase activity [27], and the reduced hydroxylysine glycosylation as a result of PLOD3 mutation is likely to reflect loss of LH3 protein, provided that other proteins, such as ColGalT1 and ColGalT2 (previously known as GLT25D1 and GLT25D2), do not significantly contribute to the glycosylation reaction.

In summary, our study demonstrates the consequences of a homozygous PLOD3 mutation in a patient with extensive connective tissue abnormalities, including skin fragility with RDEB-like phenotype. Histopathology and TEM revealed sub-lamina densa blistering, and the anchoring fibrils were perturbed with deficiency in type VII collagen. In addition to skin blistering, there was evidence of fibrosis in the upper papillary dermis, a feature of RDEB [28, 29]. It should be emphasized the WES did not reveal pathogenic mutations in COL7A1, and there was no ROH associated with chromosome 3 which harbors the COL7A1 locus. While this study was focusing on blistering phenotype and type VII collagen in this complex patient, it should be noted that all collagens undergo post-translational modifications, including glycosylation of hydroxylysine residues, and defective post-translational modifications of other members of the collagen family, such as type I, III, V and VI, are likely to explain the widespread musculoskeletal findings in these patients with complex phenotype. The physiological role of glycosylated hydroxylysine specifically in type VII collagen is not clear. One could speculate that deficient glycosylation of type VII collagen may interfere with the extracellular assembly of the anchoring fibrils or compromise their stability rendering them subject to degradation. We also noted that expression of type XVII collagen at the basement membrane zone of our patient's skin was somewhat attenuated, possibly reflecting consequences of under-glycosylation of this collagen as well. However, expression of type IV collagen, the type particularly rich in glycosylated hydroxylysine residues [30,31], was essentially normal in our patient's skin. This is 
at variance with observations in studies examining LH3 knock-out mouse embryos and embryonic fibroblasts which revealed that loss of hydroxylysine glycosylation prevents the assembly and secretion of type IV and VI collagens, with deleterious changes in the deposition and organization of the extracellular matrix [15]. Collectively, these findings, together with a previous publication of similar cases [16], indicate that a novel gene, PLOD3, can harbor mutations in the dystrophic forms of EB, and adds this gene to the growing list of candidate genes associated with skin fragility disorders in the spectrum of EB.

\section{MATERIALS AND METHODS}

\section{Patient data}

This study was approved by the Institutional Review Board of the Pasteur Institute of Iran, and all subjects and the parents of children gave written informed consent to participate in research and to publish their image. Originally, 362 extended families were included for mutational analysis. Criterion for study inclusion was a tentative diagnosis of epidermolysis bullosa (EB) based on history of neonatal cutaneous blistering suggestive of a genetic blistering disorder. One patient was found to have a trauma-induced blistering with clinical impression of dystrophic EB in addition to other systemic manifestations as described in this study.

\section{Whole exome sequencing}

DNA was extracted from peripheral blood lymphocytes of the proband by salting out method. DNA concentration was measured using a Qubit 3.0 fluorometer (Life Technologies, Carlsbad, CA, USA). The qualified genomic DNA samples were randomly fragmented by Covaris and the size of the library fragments was distributed predominantly between 150 to 200 base pairs (bp). Adapters were then ligated to both ends of the resulting fragments. The adapter-ligated templates 
were purified by the Agencourt AMPure SPRI beads and fragments with insert size about $176 \mathrm{bp}$ were excised. Extracted DNA was amplified by ligation-mediated PCR (LM-PCR), purified, and hybridized to the SureSelect Biotinylated RNA Library (BAITS) for enrichment. The hybridized fragments were bound to strepavidin beads whereas non-hybridized fragments were washed out after 24 h. Captured LM-PCR products were subjected to Agilent 2100 Bioanalyzer to estimate the magnitude of enrichment. Each captured library was then loaded on HiSeq2000 platform, and high-throughput sequencing for each captured library was performed to ensure that each sample met the desired average sequencing depth.

Raw image files were processed by Illumina Software 1.7 for base-calling with default parameters and the sequences of each individual were generated as 90/100 bp pair-end reads. The bioinformatics analysis was applied to the sequencing data ("raw data") which was generated from the Illumina pipeline. First, the adapter sequence in the raw data was removed, and low-quality reads with too many uncertain nucleotide reads or low base quality were discarded. This step produced the "clean data". Secondly, Burrows-Wheeler Aligner (BWA) was used for alignment. Read groups were added, duplicates were marked and the reads were sorted using Picard (http://broadinstitute.github.io/picard). After these processes, the final BAM files were used for variant calling using the GATK haplotype caller to identify both single nucleotide variants (SNVs) and indels. Candidate variants were then annotated using ANNOVAR software. ANNOVAR MetaSVM scores, based on several predictive bioinformatics tools, including SIFT, PolyPhen2, MutationTaster and MutationAssessor, were used to identify variants as deleterious.

\section{Variant interpretation}

SNVs and copy number variants (CNVs) were examined using the annotated CSV files of WES from the proband. The sequence variants were filtered from the VCF files for missense, 
nonsense, and splice site-affecting variants. Indel variants were filtered for exonic in-frame insertions and deletions, frameshift mutations, and gained or lost start or stop codon. Additionally, only variants with 1000 Genomes database total frequencies of $<0.001$, or those without frequency data available, were examined. These variants were then overlapped with runs of homozygosity $(\mathrm{ROH})$ in the patient. Considering the given phenotype, segregation analysis in the family, and the presence of several $\mathrm{ROH}$ in this patient with consanguineous parents, we were able to identify a likely pathogenic homozygous variant in PLOD3.

\section{Homozygosity mapping}

Whole genome-wide single nucleotide polymorphism (SNP)-based homozygosity mapping was performed by Infinium ${ }^{\circledR}$ Global Screening Array-24 v1.0 Kit ( 640,000 SNP markers) using 200 ng of genomic DNA as described by the manufacturer (Illumina,). The SNP genotyping data were analyzed by using PLINK (http://pngu.mgh.harvard.edu/). The Runs of Homozygosity algorithm in PLINK was used to identify ROH. The PLINK default values are appropriate for finding large segments of $\mathrm{ROH}$ present on dense genotyping platforms and were unchanged during the analysis. The PLINK output was filtered to eliminate $\mathrm{ROH}$ less than $4 \mathrm{Mb}$. $\mathrm{ROH}$ were compared in proband and his parents, and only those $\mathrm{ROH}$ present in the affected patient but not in the parents were considered as probable $\mathrm{ROH}$ harboring causal mutations. (For technical details of HM, see $[32,33])$

\section{Cell culture and LH3 expression analysis:}

Primary dermal fibroblast cultures of the patient and controls were established from a skin biopsy and were grown under standard conditions including DMEM (Dulbecco's Modified Eagle Medium) and 10\% fetal bovine serum (FBS). Total RNA was extracted in triplicate from cultured cells derived from the patient and controls using the RNeasy mini kit (Qiagen, Hilden, 
Germany) with on-column DNase digestion (Qiagen) according to the manufacturer's instructions. cDNA was synthesized from $1 \mu \mathrm{g}$ of mRNA using the iScript cDNA synthesis kit (Bio-Rad Laboratories, Hercules, CA, USA), followed by reverse transcription quantitative realtime PCR (RT-qPCR) amplification. The expression level was determined for the PLOD3 gene using the reference genes HPRTI, YWHAZ, and RPL13A for normalization (primers and conditions available on request). Each sample was analysed in duplicate and data analysis was performed with the qBasePlus software (Biogazelle, Ghent, Belgium).

\section{Transcriptome profiling of collagen gene expression by heatmap analysis}

Total RNA was extracted from the fibroblast obtained from the patient and a healthy controls using TRIzol@ Reagent and quantified on a Nanodrop ND-100 spectrophotometer (Thermo Fisher Scientific, Wilmington, DE), followed by RNA quality assessment on an Agilent 2200 TapeStation (Agilent Technologies, Palo Alto, CA). Multiplexed library construction, workflow analysis, and sequencing runs were performed following standard Illumina protocols (Illumina, Inc., San Diego, CA) using the TruSeq Stranded Total RNA kit with indexes from Set A (Cat \#: RS-122-2301). Paired-end $(2 \times 75)$ sequencing reads were generated on the Illumina NextSeq 500 platform and stored in FASTQ format. FASTQ quality was checked using FastQC, and the TruSeq RNA adapter sequences were removed by Trimmomatic. Alignment and mapping were performed using STAR-2pass (v. 2.5.3a) [34] with the human reference genome (GRCh38/hg38) and GENCODE V27 annotations. In the first pass, an initial alignment was executed and the splice junction information was collected. This information was then used for the second pass in which the final alignment was performed. The alignments were sorted, read group was added, and the corresponding index was created (using Picard tools).

\section{Western blotting}


Cell lysates were collected from dermal fibroblast cultures using RIPA lysis buffer (SigmaAldrich) complemented with protease (Roche) and phosphatase (Sigma-Aldrich) inhibitors and subjected to SDS-PAGE on 4-12\% Bis-Tris gel (Invitrogen, Thermo Fisher Scientific).

Subsequently, proteins were transferred to a nitrocellulose membrane using the iBlot System (Thermo Fisher Scientific) and immunolabeled with PLOD3 Rabbit Polyclonal antibody (1:500, Proteintech Europe, Manchester, United Kingdom) and horseradish peroxidase (HRP) conjugated secondary antibody (Cell Signaling Technologies, Danvers, MA, USA). Membranes were developed using the SuperSignal West Dura Extended Duration Substrate kit (Thermo Fisher Scientific, Life Technologies Europe, Ghent, Belgium) and scanned with the Amersham Imager 680 (GE Healthcare Life Sciences). Following stripping, the membrane was reprobed with $\beta$-tubulin (Abcam, Cambridge, United Kingdom) in order to check for equal loading. This analysis was repeated three times. Intensity of the bands was quantified using the ImageJ software.

\section{Measurements of urinary hydroxylysine derivatives}

Urine samples were collected from proband and his parents. Urine samples and isolated peptide fractions were acid hydrolyzed and analyzed by reverse phase HPLC as previously described (Kraenzlin et al., 2008). To allow for the detection of galactosyl-hydroxylysylpyridinoline (GHL) and glucosyl-galactosyl-hydroxylysine (GGHL), as pyridinoline compounds derived from collagen crosslinks, samples were analyzed also without prior acid hydrolysis.

\section{Immunofluorescence staining}

Skin sections $(5 \mu \mathrm{m})$ were air-dried and initially blocked with diluted normal goat serum (SigmaAldrich, Dorset, UK), and then incubated with anti-LH3 antibody diluted 1:10 in phosphatebuffered saline with 30\% w/v bovine serum albumin (Sigma-Aldrich). After washing in 
phosphate-buffered saline, slides were labeled with fluorescein isothiocyanate secondary antibodies (Invitrogen, Paisley, UK). Negative controls omitting the primary antibody were also performed. All sections were photographed using the same camera and identical exposure times (3 seconds). A list of antibodies used for characterization of the skin from the proband with homozygous PLOD3 mutation can be found in Supplementary Table S3.

\section{Mean Fluorescence Intensity}

Mean Fluorescence Intensity (MFI) was calculated for each IF section using Image J (http://rsb.info.nih.gov/ij/). Ten measurements were taken at regular intervals using $8 \times 8$ pixels every 100 pixel along the dermal-epidermal junction. The values are presented as mean \pm standard error of mean (SE) as well as a percent of the staining intensity in the skin of a healthy, unrelated control (100\%).

\section{Transmission electron microscopy}

Skin biopsy specimens were cut into small pieces $\left(<1 \mathrm{~mm}^{3}\right)$ and fixed in half-strength Karnovsky fixative for 4 hours at room temperature. After washing in $0.1 \mathrm{M}$ phosphate buffer $(\mathrm{pH} 7.4)$, the samples were immersed in $1.3 \%$ aqueous osmium tetroxide (TAAB Laboratories, Berkshire, UK) for 2 hours, followed by incubation in 2\% uranyl acetate (Bio-Rad, Hertfordshire, UK), and dehydrated in a graded ethanol series, and then embedded in epoxy resin via propylene oxide (TAAB Laboratories). Ultra-thin sections were stained with uranyl acetate and lead citrate and examined in a Philips CM10 transmission electron microscope (Philips, Eindhoven, The Netherlands).

\section{LH3 protein modeling}

Three-dimensional structure of LH3 was adopted from a published study [35] (PDB entry: 6FXR). Molecular graphics were visualized using VMD version 1.9.3 [36]. 


\section{ACKNOWLEDGMENTS}

The authors thank Sara Norouz-zadeh, Niloufar Amirinezhad and Dr. Amir Hesam Saeidian for assistance in the collection and processing of the samples and the clinical data. Marine Vanhomwegen, Jozefien Weytens and Lynn Demuynck provided excellent technical assistance. Ali Jazayeri contributed to WES data analysis. Drs. Mehdi Farshchian and Andrew South provided helpful suggestions. Carol Kelly assisted in manuscript preparation. Deiflen Syx is a postdoctoral researcher, and Fransiska Malfait is a senior clinical researcher, both supported by the Research Foundation Flanders, Belgium (FWO). This work was supported by a Methusalem Grant from Ghent University to Anne De Paepe (BOFMET2015000401) and by DEBRA International (JU). This study is in partial fulfillment of the PhD Thesis of HV. 


\section{References}

[1] J.D. Fine, L. Bruckner-Tuderman, R.A. Eady, E.A. Bauer, J.W. Bauer, C. Has, A. Heagerty, H. Hintner, A. Hovnanian, M.F. Jonkman, I. Leigh, M.P. Marinkovich, A.E. Martinez, J.A. McGrath, J.E. Mellerio, C. Moss, D.F. Murrell, H. Shimizu, J. Uitto, D. Woodley, G. Zambruno, Inherited epidermolysis bullosa: updated recommendations on diagnosis and classification. J Am Acad Dermatol 70(6) (2014) 1103-1126.

[2] J. Uitto, C. Has, H. Vahidnezhad, L. Youssefian, L. Bruckner-Tuderman, Molecular pathology of the basement membrane zone in heritable blistering diseases: The paradigm of epidermolysis bullosa. Matrix Biol 57-58 (2017) 76-85.

[3] C. Has, A. Nystrom, A.H. Saeidian, L. Bruckner-Tuderman, J. Uitto, Epidermolysis bullosa: Molecular pathology of connective tissue components in the cutaneous basement membrane zone. Matrix Biol 71-72 (2018) 313-329.

[4] R.V. Iozzo, M.A. Gubbiotti, Extracellular matrix: The driving force of mammalian diseases. Matrix Biol 71-72 (2018) 1-9.

[5] H. Vahidnezhad, L. Youssefian, A.H. Saeidian, H.R. Mahmoudi, A. Touati, M. Abiri, A.M. Kajbafzadeh, S. Aristodemou, L. Liu, J.A. McGrath, A. Ertel, E. Londin, A. Kariminejad, S. Zeinali, P. Fortina, J. Uitto, Recessive mutation in tetraspanin CD151 causes Kindler syndromelike epidermolysis bullosa with multi-systemic manifestations including nephropathy. Matrix

Biol 66

(2018) 22-33.

[6] J.E. Mellerio, S.J. Robertson, C. Bernardis, A. Diem, J.D. Fine, R. George, D. Goldberg, G.B. Halmos, M. Harries, M.F. Jonkman, A. Lucky, A.E. Martinez, E. Maubec, S. Morris, D.F. Murrell, F. Palisson, E.I. Pillay, A. Robson, J.C. Salas-Alanis, J.A. McGrath, Management of 
cutaneous squamous cell carcinoma in patients with epidermolysis bullosa: best clinical practice guidelines. Br J Dermatol 174(1) (2016) 56-67.

[7] H.J. Chung, J. Uitto, Type VII collagen: the anchoring fibril protein at fault in dystrophic epidermolysis bullosa. Dermatol Clin 28(1) (2010) 93-105.

[8] L.Y. Sakai, D.R. Keene, N.P. Morris, R.E. Burgeson, Type VII collagen is a major structural component of anchoring fibrils. J Cell Biol 103(4) (1986) 1577-86.

[9] D. Villone, A. Fritsch, M. Koch, L. Bruckner-Tuderman, U. Hansen, P. Bruckner, Supramolecular interactions in the dermo-epidermal junction zone: anchoring fibril-collagen VII tightly binds to banded collagen fibrils. J Biol Chem 283(36) (2008) 24506-13.

[10] A.M. Salo, L. Sipila, R. Sormunen, H. Ruotsalainen, S. Vainio, R. Myllyla, The lysyl hydroxylase isoforms are widely expressed during mouse embryogenesis, but obtain tissue- and cell-specific patterns in the adult. Matrix Biol 25(8) (2006) 475-83.

[11] K.I. Kivirikko, T. Pihlajaniemi, Collagen hydroxylases and the protein disulfide isomerase subunit of prolyl 4-hydroxylases. Adv Enzymol Relat Areas Mol Biol 72 (1998) 325-98.

[12] R. Myllyla, C. Wang, J. Heikkinen, A. Juffer, O. Lampela, M. Risteli, H. Ruotsalainen, A. Salo, L. Sipila, Expanding the lysyl hydroxylase toolbox: new insights into the localization and activities of lysyl hydroxylase 3 (LH3). J Cell Physiol 212(2) (2007) 323-9.

[13] C. Wang, M.M. Ristiluoma, A.M. Salo, S. Eskelinen, R. Myllyla, Lysyl hydroxylase 3 is secreted from cells by two pathways. J Cell Physiol 227(2) (2012) 668-75.

[14] S.A. Watt, J.H. Dayal, S. Wright, M. Riddle, C. Pourreyron, J.R. McMillan, R.M. Kimble, M. Prisco, U. Gartner, E. Warbrick, W.H. McLean, I.M. Leigh, J.A. McGrath, J.C. Salas-Alanis, J. Tolar, A.P. South, Lysyl hydroxylase 3 localizes to epidermal basement membrane and is 
reduced in patients with recessive dystrophic epidermolysis bullosa. PLoS One 10(9) (2015) e0137639.

[15] M. Risteli, H. Ruotsalainen, A.M. Salo, R. Sormunen, L. Sipila, N.L. Baker, S.R. Lamande, L. Vimpari-Kauppinen, R. Myllyla, Reduction of lysyl hydroxylase 3 causes deleterious changes in the deposition and organization of extracellular matrix. J Biol Chem 284(41) (2009) 28204-11. [16] A.M. Salo, H. Cox, P. Farndon, C. Moss, H. Grindulis, M. Risteli, S.P. Robins, R. Myllyla, A connective tissue disorder caused by mutations of the lysyl hydroxylase 3 gene. Am J Hum Genet 83(4) (2008) 495-503.

[17] H. Vahidnezhad, L. Youssefian, A.H. Saeidian, N. Mozafari, M. Barzegar, S. Sotoudeh, M. Daneshpazhooh, A. Isaian, S. Zeinali, J. Uitto, KRT5 and KRT14 mutations in epidermolysis bullosa simplex with phenotypic heterogeneity, and evidence of semidominant inheritance in a multiplex family. J Invest Dermatol 136(9) (2016) 1897-901.

[18] H. Vahidnezhad, L. Youssefian, A.H. Saeidian, A. Touati, S. Sotoudeh, M. Abiri, M. Barzgar, N. Aghazadeh, H. Mahmoudi, S. Norouz-zadeh, M. Hamid, M. Zahabiyon, H. Bagherian, S. Zeinali, P. Fortina, J. Uitto, Multigene next generation sequencing panel identifies pathogenic variants in patients with unknown subtype of epidermolysis bullosa: Subclassification with prognostic implications. J Invest Dermatol 137 (2017) 2649-2652.

[19] H. Vahidnezhad, L. Youssefian, S. Zeinali, A.H. Saeidian, S. Sotoudeh, N. Mozafari, M. Abiri, A.M. Kajbafzadeh, M. Barzegar, A. Ertel, P. Fortina, J. Uitto, Dystrophic epidermolysis bullosa: COL7A1 mutation landscape in a multi-ethnic cohort of 152 extended families with high degree of customary consanguineous marriages. J Invest Dermatol 137(3) (2017) 660-669. [20] H. Vahidnezhad, L. Youssefian, A.H. Saeidian, A. Touati, S. Sotoudeh, A. Jazayeri, A. Guy, P.A. Lovell, L. Liu, A. Kariminejad, J.A. McGrath, S. Zeinali, J. Uitto, Next generation 
sequencing identifies double homozygous mutations in two distinct genes (EXPH5 and COL17A1) in a patient with concomitant simplex and junctional epidermolysis bullosa. Hum Mutat 39 (2018) 1349-1354.

[21] K. Wang, M. Li, H. Hakonarson, ANNOVAR: functional annotation of genetic variants from high-throughput sequencing data. Nucleic Acids Res 38(16) (2010) e164.

[22] T. Wong, L. Gammon, L. Liu, J.E. Mellerio, P.J. Dopping-Hepenstal, J. Pacy, G. Elia, R. Jeffery, I.M. Leigh, H. Navsaria, J.A. McGrath, Potential of fibroblast cell therapy for recessive dystrophic epidermolysis bullosa. J Invest Dermatol 128(9) (2008) 2179-89.

[23] E.R. Savolainen, M. Kero, T. Pihlajaniemi, K.I. Kivirikko, Deficiency of galactosylhydroxylysyl glucosyltransferase, an enzyme of collagen synthesis, in a family with dominant epidermolysis bullosa simplex. N Engl J Med 304(4) (1981) 197-204.

[24] J.D. Fine, R.D. Griffith, A specific defect in glycosylation of epidermal cell membranes. Definition in skin from patients with epidermolysis bullosa simplex. Arch Dermatol 121(10) (1985) 1292-6.

[25] C. Has, G. Sparta, D. Kiritsi, L. Weibel, A. Moeller, V. Vega-Warner, A. Waters, Y. He, Y. Anikster, P. Esser, B.K. Straub, I. Hausser, D. Bockenhauer, B. Dekel, F. Hildebrandt, L. Bruckner-Tuderman, G.F. Laube, Integrin alpha3 mutations with kidney, lung, and skin disease. N Engl J Med 366(16) (2012) 1508-14.

[26] H. Vahidnezhad, L. Youssefian, A.H. Saeidian, J. Uitto, Phenotypic spectrum of epidermolysis bullosa, the paradigm of syndromic versus non-syndromic skin fragility disorders. J Invest Dermatol (in press) (2018).

[27] B. Schegg, A.J. Hulsmeier, C. Rutschmann, C. Maag, T. Hennet, Core glycosylation of collagen is initiated by two beta(1-O)galactosyltransferases. Mol Cell Biol 29(4) (2009) 943-52. 
[28] A. Nystrom, L. Bruckner-Tuderman, Injury- and inflammation-driven skin fibrosis: The paradigm of epidermolysis bullosa. Matrix Biol 68-69 (2018) 547-560.

[29] S. Ricard-Blum, G. Baffet, N. Theret, Molecular and tissue alterations of collagens in fibrosis. Matrix Biol 68-69 (2018) 122-149.

[30] S.D. Funk, M.H. Lin, J.H. Miner, Alport syndrome and Pierson syndrome: Diseases of the glomerular basement membrane. Matrix Biol 71-72 (2018) 250-261.

[31] K.I. Kivirikko, R. Myllyla, Collagen glycosyltransferases. Int Rev Connect Tissue Res 8 (1979) 23-72.

[32] H. Vahidnezhad, L. Youssefian, A.H. Saeidian, S. Zeinali, M. Abiri, S. Sotoudeh, S. Norouz-zadeh, N. Amirinezhad, N. Mozafari, N. Aghazadeh, H. Mahmoudi, J.P. Bradford, C.E. Kim, H. Hakonarson, J. Uitto, Genome-wide single nucleotide polymorphism-based autozygosity mapping facilitates identification of mutations in consanguineous families with epidermolysis bullosa. Exp Derm 138 (2018) 121-131.

[33] H. Vahidnezhad, L. Youssefian, A. Jazayeri, J. Uitto, Research techniques made simple: Genome-wide homozygosity/autozygosity mapping is a powerful tool to identify candidate genes in autosomal recessive genetic diseases. J Invest Dermatol 138 (2018) 1893-1900.

[34] A. Dobin, C.A. Davis, F. Schlesinger, J. Drenkow, C. Zaleski, S. Jha, P. Batut, M. Chaisson, T.R. Gingeras, STAR: Ultrafast universal RNA-seq aligner. Bioinformatics 29(1) (2013) 15-21. [35] L. Scietti, A. Chiapparino, F. De Giorgi, M. Fumagalli, L. Khoriauli, S. Nergadze, S. Basu, V. Olieric, L. Cucca, B. Banushi, A. Profumo, E. Giulotto, P. Gissen, F. Forneris, Molecular architecture of the multifunctional collagen lysyl hydroxylase and glycosyltransferase LH3. Nat Commun 9(1) (2018) 3163. 
[36] W. Humphrey, A. Dalke, K. Schulten, VMD: visual molecular dynamics. J Mol Graph 14(1) (1996) 33-8, 27-8. 


\section{Figure Legend}

Figure 1. Family pedigree and clinical features of the proband. (a) The proband (arrowhead) was born to clinically healthy parents who are first cousins once removed. (b, c) Note the presence of a tense, hemorrhagic blister on the fifth toe and small erosions on arm (white arrows) with evidence of atrophic scarring at the elbow. (d) Chest X-ray demonstrating the diaphragmatic eventration. (e, f) Presence of contractions on the elbow and the digits on the left hand. (g, h). Presence of severe scoliosis noted clinically and confirmed by MRI.

Figure 2. Identification of homozygous PLOD3 mutation. (a) Homozygosity mapping revealed a $\sim 45 \mathrm{Mb}$ region of homozygosity in the proband ( $\mathrm{V}-2)$, which was not present in the parents (IV1, IV-2); this region on chromosome 7 contained the locus for PLOD3. (b) Whole exome sequencing identified a total of $\sim 134,000$ annotated sequence variants which were filtered by steps indicated to yield 16 variants with minor allelic frequency (MAF) of $<1: 1,000$. Eight of these variants were within the different regions of homozygosity as shown in (a), and matching the phenotype of the patient identified a mutation in PLOD3. The homozygous mutation in the patient, p.Leu627Pro, was confirmed by Sanger sequencing (right upper panel) and the parents were heterozygous carriers of the mutation (lower panel). (d) Three-dimensional structure of LH3. The p.Leu627Pro mutation within the lysyl hydroxylase (LH) domain is presented in red. Previously reported p.Asn223Ser missense mutation in glycosyltransferase (GT) domain is depicted in purple and frameshift mutation (p.Cys691AlafsX9) results in deletion of 40 amino acid residues at the end of C-terminus of the protein colored in yellow. Ferrous iron molecules are shown as brown spheres, and 2-oxoglutarate as black CPK representation. (e and f) Western blotting revealed markedly reduced LH3 protein levels in fibroblasts cultured from the skin of 
the patient $(\mathrm{P})$ as compared to controls (C1-3). Reprobing the filter with an anti- $\beta$-tubulin antibody revealed equal protein loading. The LH3 protein levels were quantitated by scanning the bands in Western blots in three separate experiments (mean $\pm \mathrm{SEM} ;{ }^{* *} \mathrm{p}<0.01, * * * \mathrm{p}<0.001$ ). (g) Heatmap based transcriptome expression profiling of fibroblast obtained from patient revealed significantly reduced COL7A1 mRNA levels in comparison to several randomly selected housekeeping genes. No significant difference was observed in gene expressions of PLOD3 and types I, IV, V, and VI collagens.

Figure 3. Demonstration of sub-lamina densa tissue separation in the skin of the proband. (a) Histopathology reveals separation at the dermal-epidermal junction (Richardson's stain). (b) Transmission electron microscopy reveals that the blister formation is below the lamina densa which is delineated by arrows. (Blister marked by an asterisk).

Figure 4. Transmission electron microscopy of patient skin reveals fragmentation of the lamina densa. (a) The lamina densa within the right half of this skin section appears intact but on the left half it is interrupted and fragmented. (b) Patchy areas of intact lamina densa are present (arrows) below which there is some sub-lamina densa cleavage (asterisks). (c) In the superficial dermis, there are fibroblasts (arrows) close to the dermal-epidermal junction that display dilated endoplasmic reticulum and prominent vesicles close to the cell membrane, with some adjacent dermal cleavage (asterisk). 
Figure 5. Demonstration of fibrosis in the upper dermis of the skin of the proband. (a)

Histopathology at lower magnification reveals a homogeneous fibrotic zone in the upper dermis.

(b) Higher magnification reveals a number of fibroblastic cells in the upper papillary dermis juxtaposed to the dermal-epidermal junction. (c) Transmission electron microscopy of the upper dermis reveals the presence of fibroblastic cells with dilated cisternae of rough endoplasmic reticulum, embedded in dense collagenous meshwork (a, b: Richardson's stain).

Figure 6. Transmission electron microscopy reveals variations in the number and morphology of anchoring fibrils of the proband's skin. (a) In some areas, anchoring fibrils with normal appearance are present (arrows). (b) In some areas, the anchoring fibrils are wispy, hypoplastic and reduced in number. (c, d) Anchoring fibrils are absent in areas of blistering (asterisks).

Figure 7. Immunofluorescence analysis and immuno-epitope mapping of the location of the blistering in the skin of the proband. (a) Immunofluorescence reveals complete absence of LH3 in the patient's skin with a blister (asterisk), while in the control skin, a punctate pattern at the dermal-epidermal junction is noted. Type VII collagen expression is significantly reduced, the remaining protein being primarily in the roof of the blister. Type IV collagen staining reveals normal amounts of protein at the dermal-epidermal junction primarily in the roof of the blister. The staining on the floor of the blister is attributable to neovascularization as part of the regenerative fibrotic process. (b) Immunofluorescence reveals attenuated intensity of staining with antibodies to type XVII collagen and plectin, while laminin-332 and $\beta 4$ integrin staining is of normal intensity and primarily in the roof of the blister. (c) Staining for different keratins 
reveals somewhat attenuated staining patterns presumably reflecting overall perturbation of the structure at the basement membrane zone. (Scale bar $=50 \mu \mathrm{m})$.

Figure 8. Demonstration of reduced type VII collagen expression in the skin of the proband by Mean Fluorescent Intensity (MFI) analysis using Image J (National Institutes of Health, Bethesda, MD). (a) Biopsies were obtained from different areas of skin and stained for type VII collagen (Images I-IV). (b) The intensity of the staining was morphometrically quantitated and calculated as a percent of the staining intensity in the skin of a healthy, unrelated control. (c) Graphical representation of MFI for type VII collagen expression in the skin of the proband compared to an unrelated control. Each bar represents the mean value of 10 measurements of type VII collagen expression intensity along the dermal-epidermal junction from corresponding images (I-IV); error bars represent standard error of the mean.

Figure 9. Analysis of the lysine (L), hydroxylysine (HL) and its glycosylated derivatives (glucosyl-galactosyl hydroxylysine, GGHL; and glucosyl hydroxylysine, GHL), analyzed in the urine of the patient and his parents as pyridinoline compounds. The peaks represent: 1, GGHL; 2 , GHL; 3, HL; 4,L; IS, internal standard in the liquid chromatography. The values were calculated from the area under each peak as a percent of total (mean of two independent measurements). 


\section{Highlights}

- Recessive dystrophic epidermolysis bullosa is caused by mutations in COL7A1

- We have evaluated a patient with multisystem disorder including blistering phenotype

- NGS identified mutations in PLOD3 encoding lysyl hydroxylase 3 (LH3)

- The mutant LH3 was associated with reduced type VII collagen and anchoring fibrils

- PLOD3 is the second mutant gene associated with RDEB 


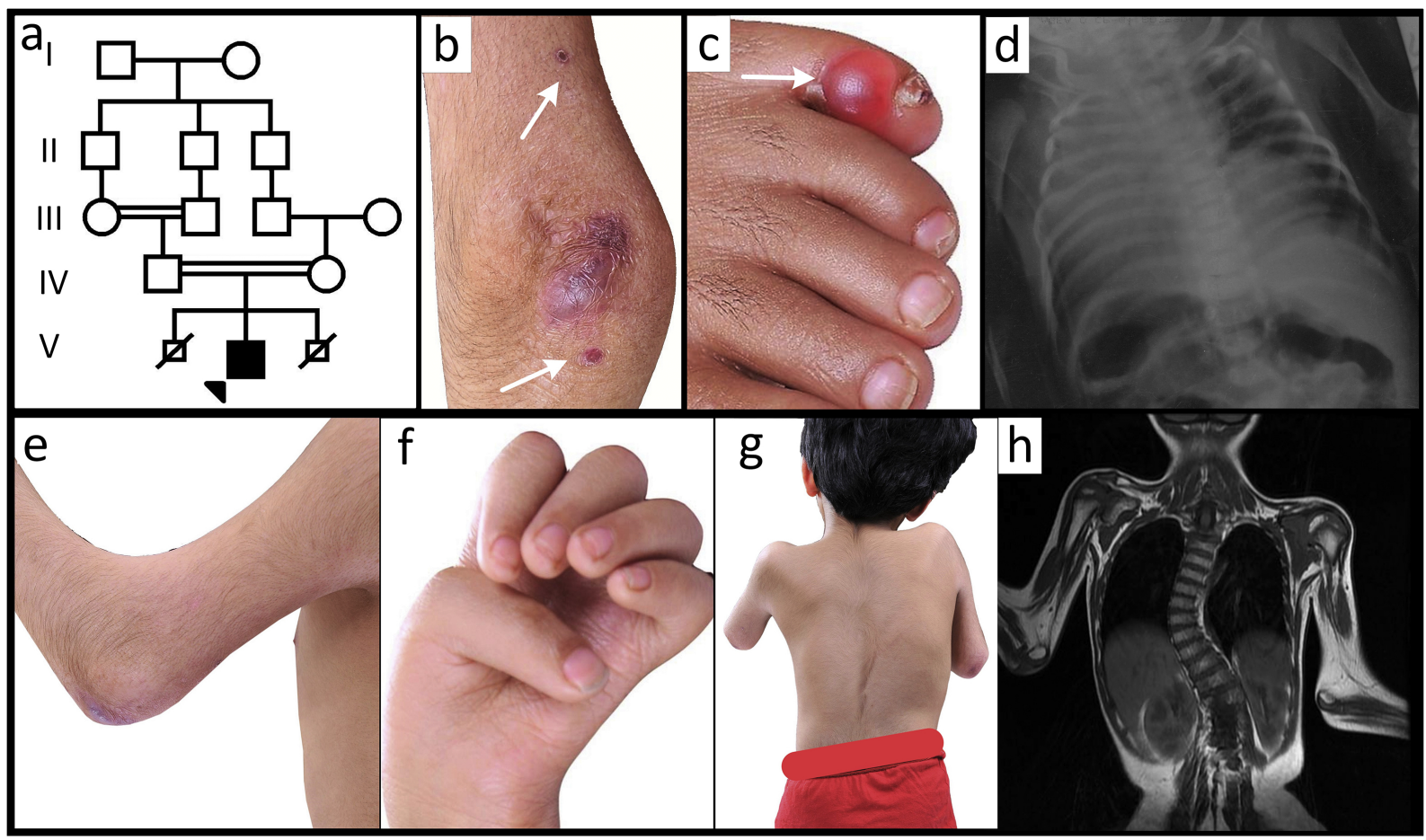

Figure 1 


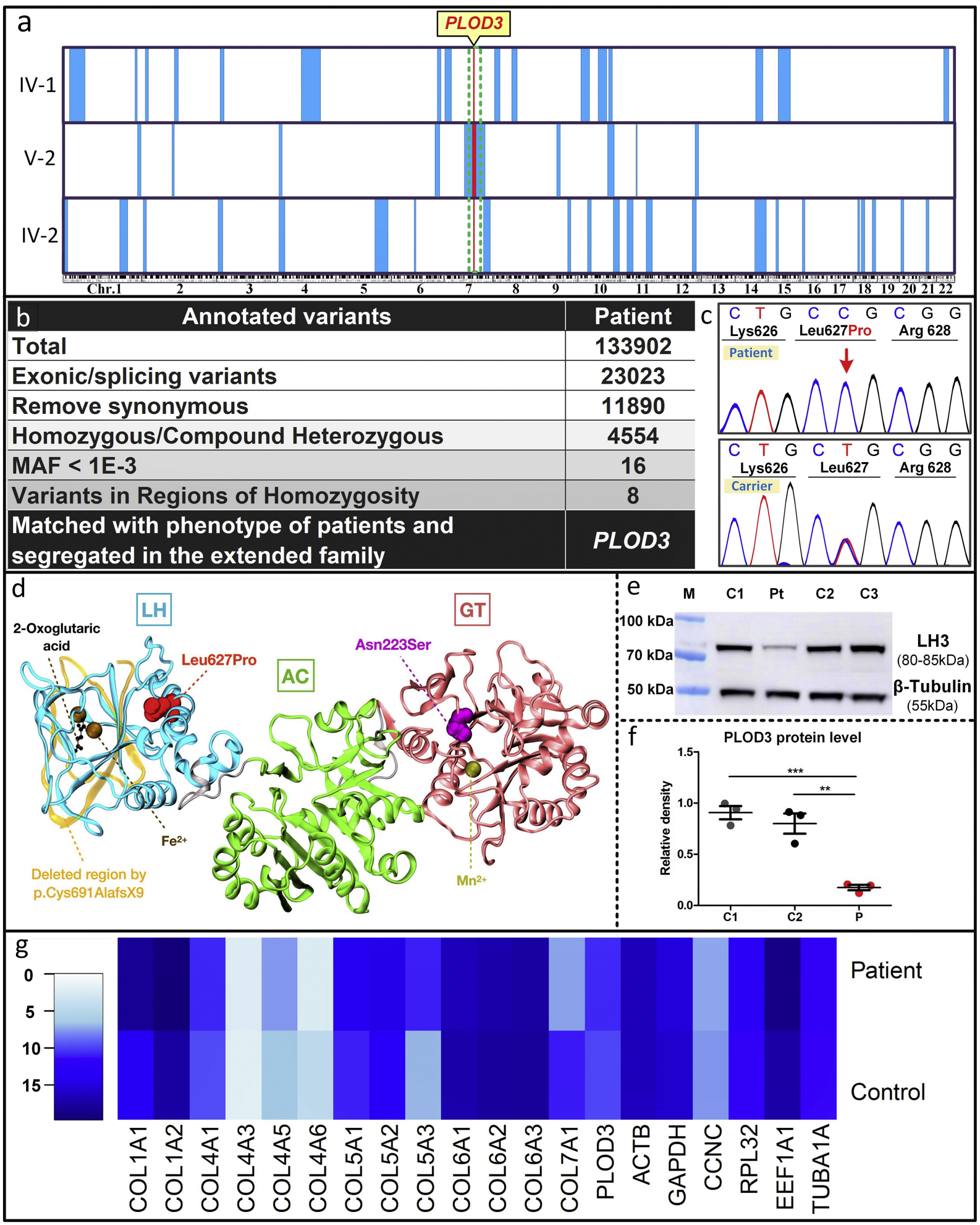




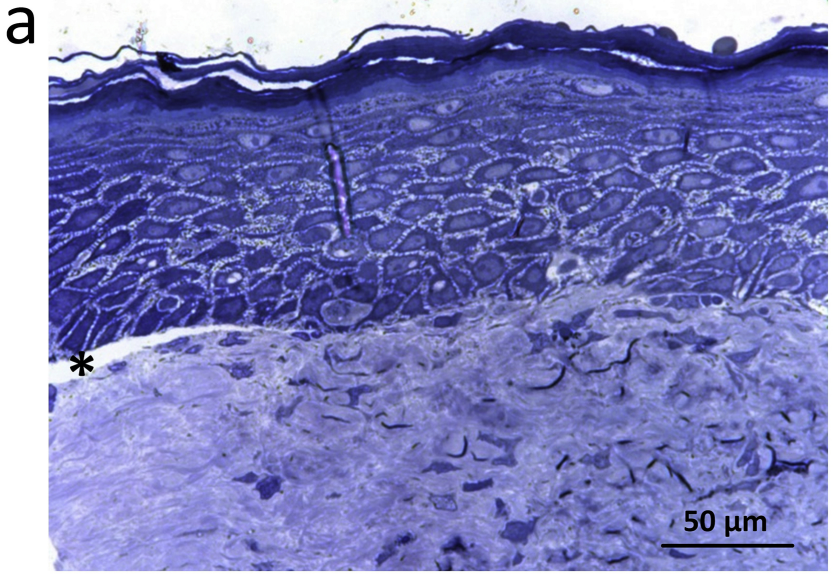

b

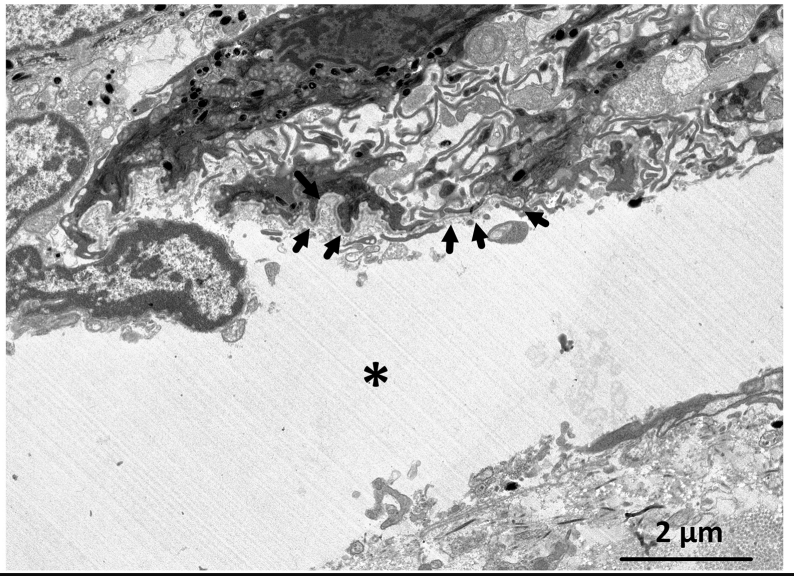

Figure 3 


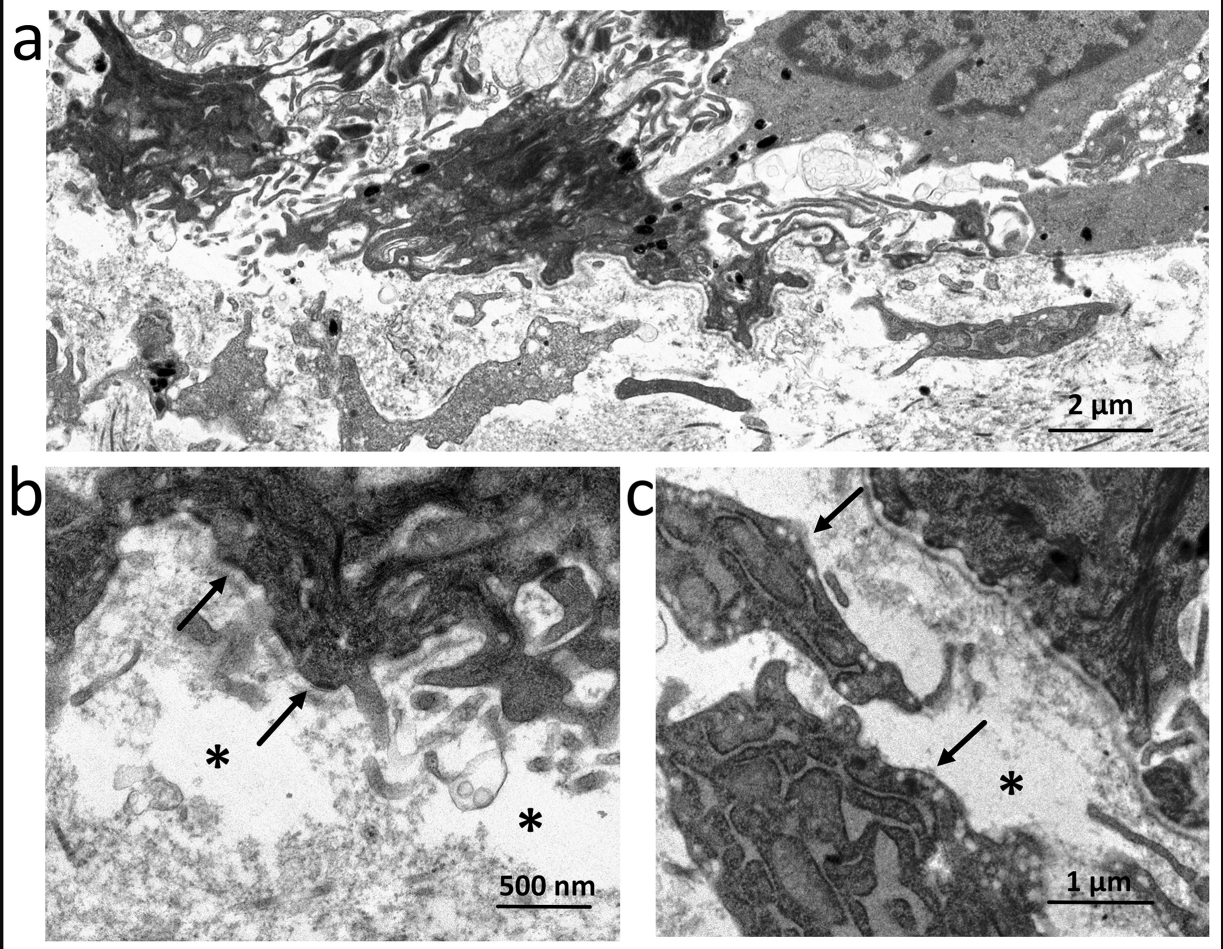

Figure 4 


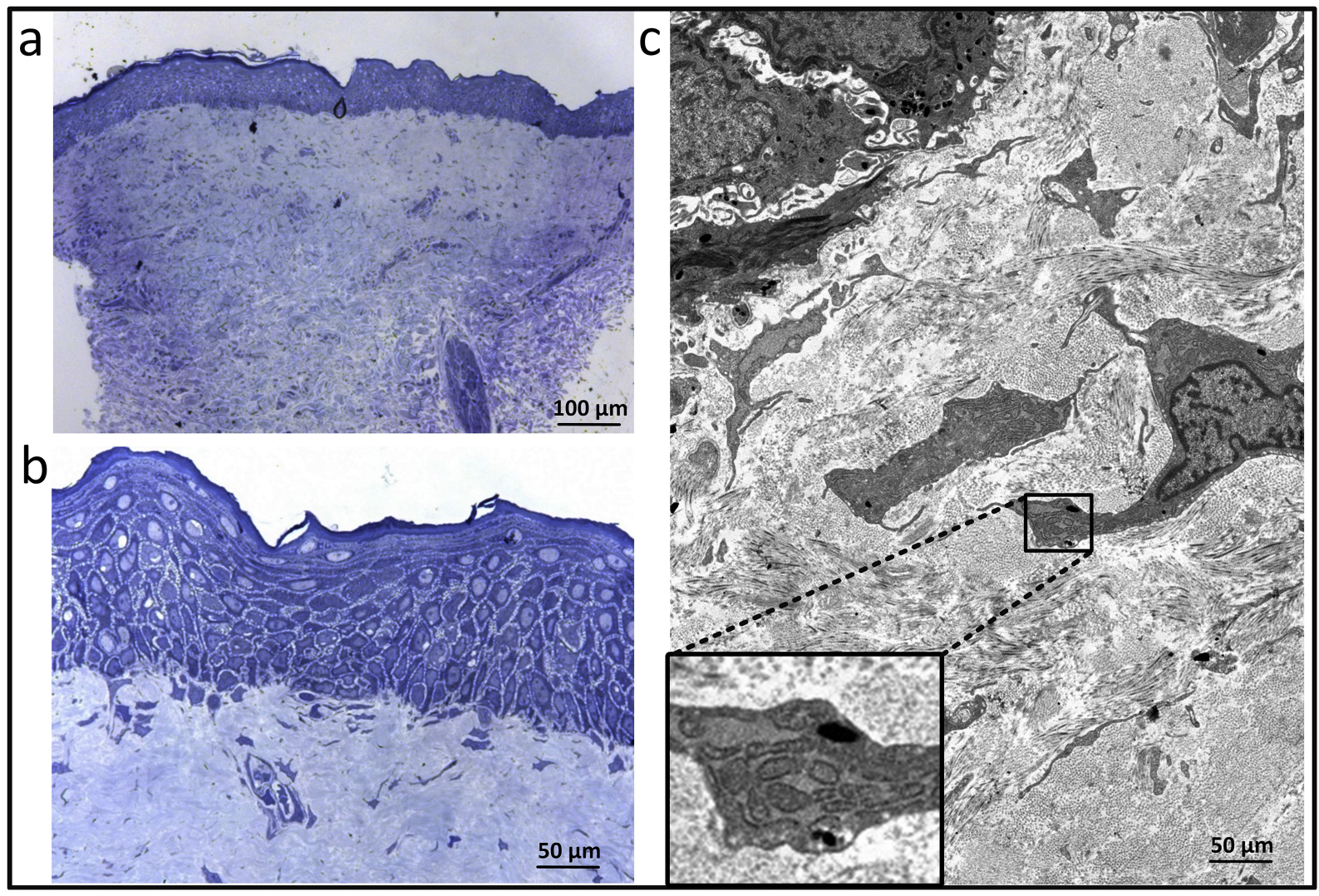

Figure 5 


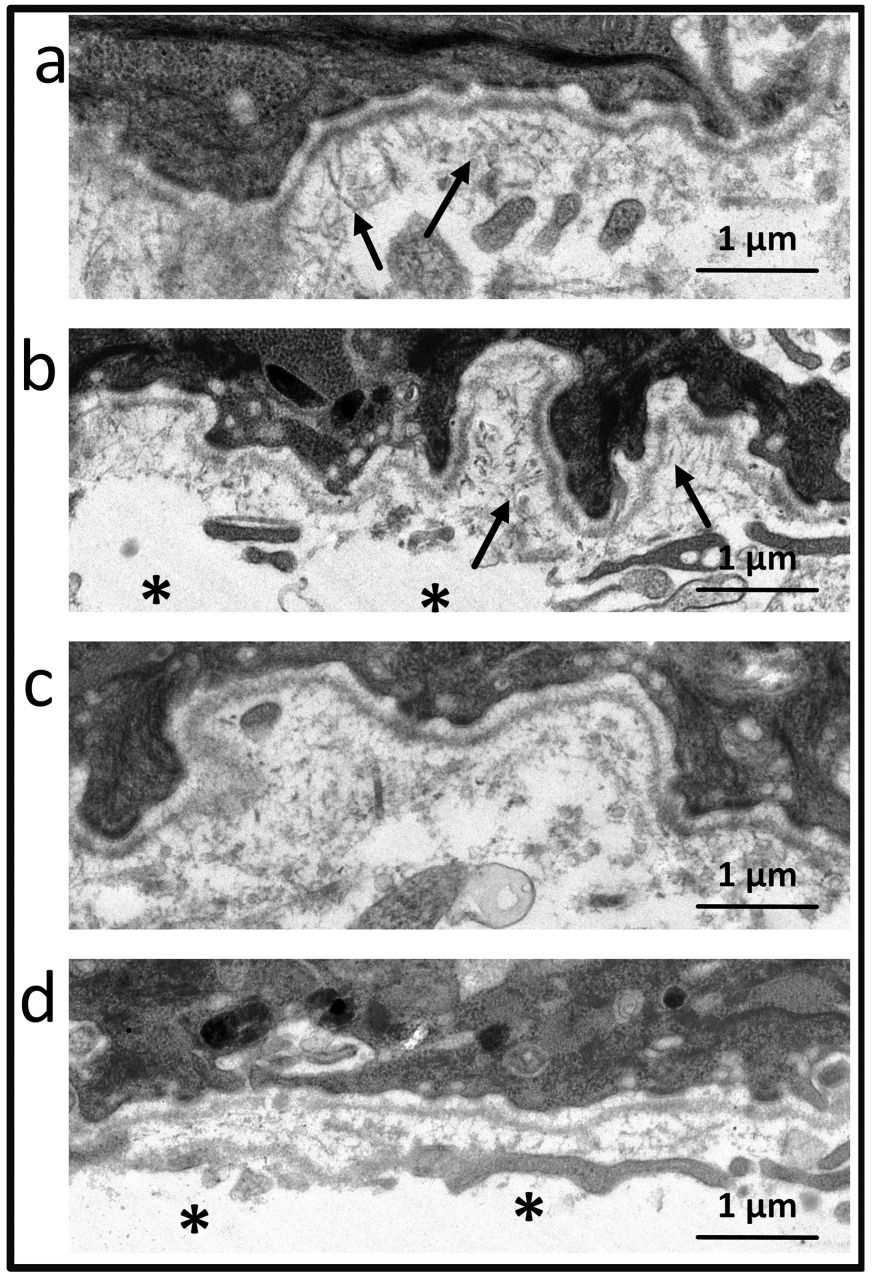

Figure 6 


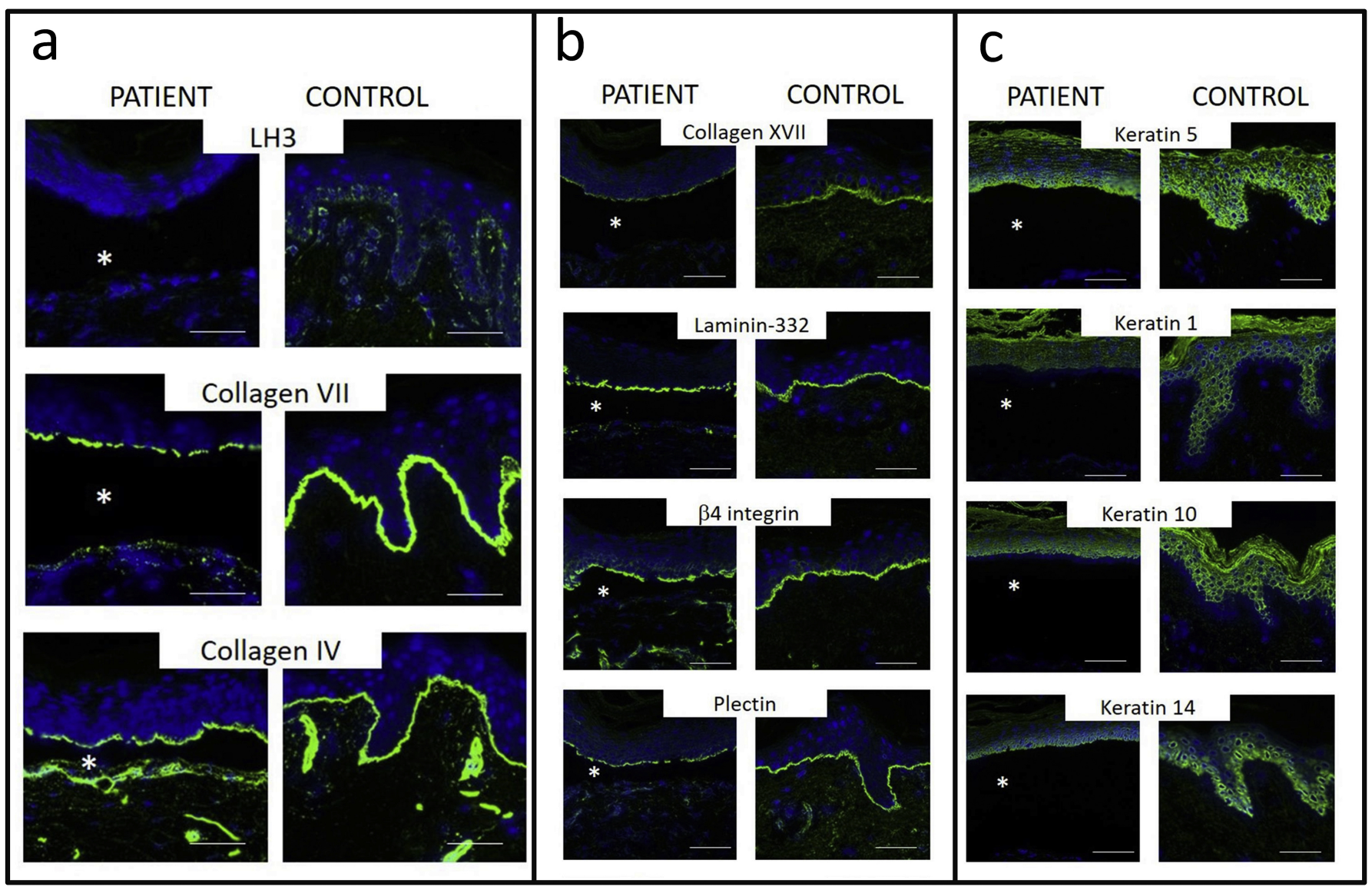

Figure 7 


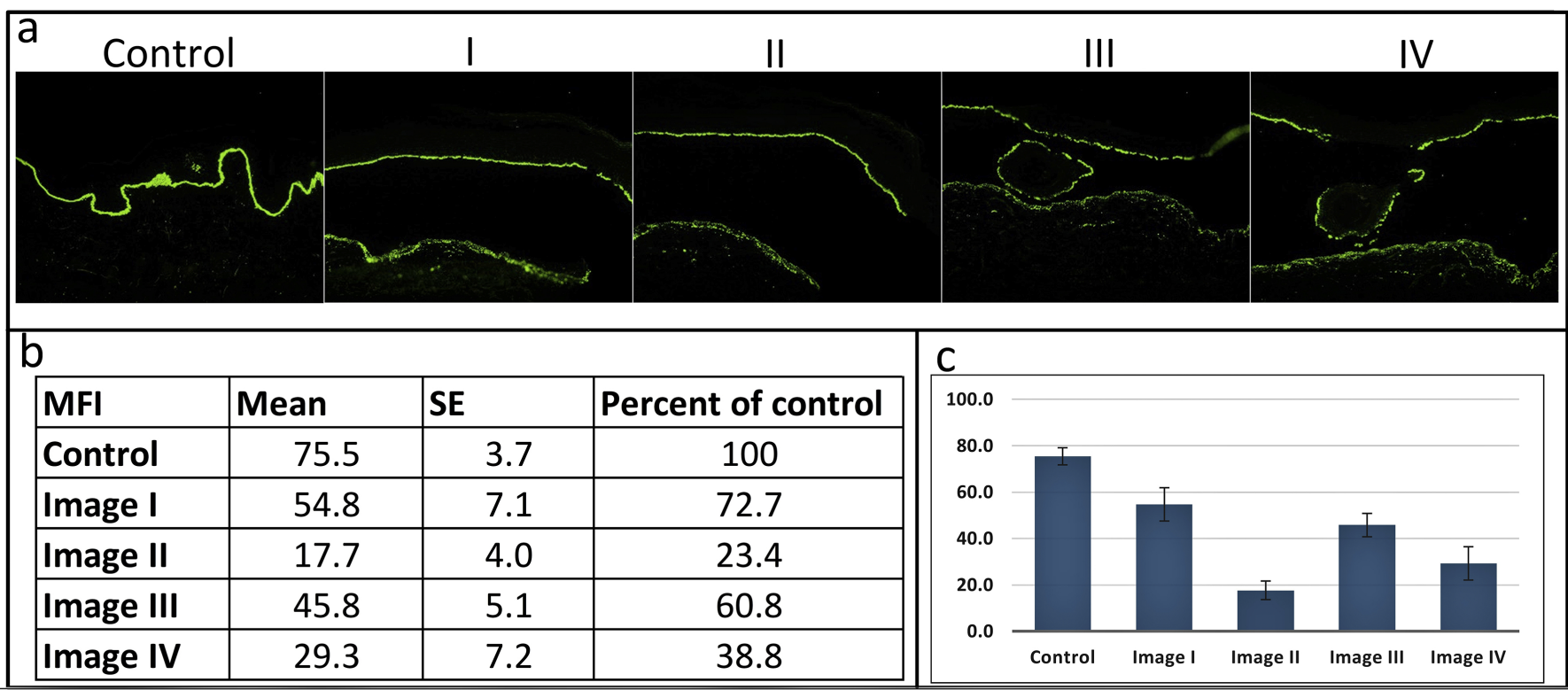

Figure 8 


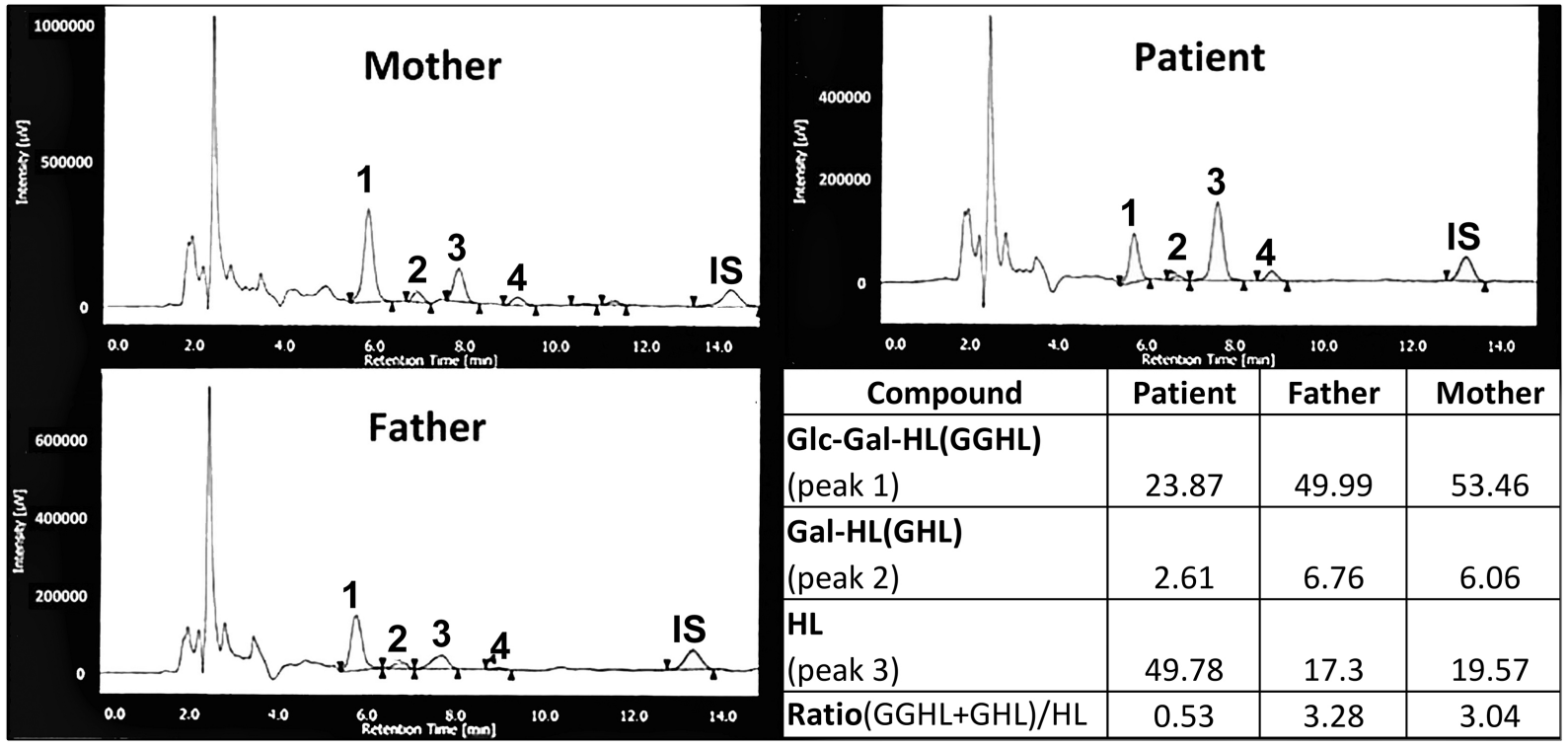

Figure 9 